\title{
Principios básicos de posicionamiento comunicativo. Análisis del caso de Tuenti (2012-2015)
}

\section{Basic principles of positioning in communication. Case analysis: Tuenti (2012-2015)}

\author{
Cristóbal Fernández Muñoz \\ Profesor asociado \\ (Universidad Complutense de Madrid) \\ Esperanza Martínez Herrero \\ Máster en Comunicación de las Organizaciones \\ (Universidad Complutense Madrid) \\ María Luisa García Guardia \\ Profesora titular \\ (Universidad Complutense Madrid)
}

Fecha de recepción: 31 de octubre de 2016

Fecha de revisión: 20 de marzo de 2017

Fecha de publicación: 1 de julio de 2017

Para citar este artículo: Fernández Muñoz, C., Martínez Herrero, E. y García Guardia, M. L. (2017): Principios básicos de posicionamiento comunicativo. Análisis del caso de Tuenti (2012-2015), Icono 14, volumen 15 (2), pp. 49-69. doi: 10.7195/ri14.v15i2.1000 


\section{ARTÍCULOS DE INVESTIGACIÓN}

\section{Resumen}

Actualmente, las empresas tienen como objetivo ser singulares y diferenciarse en un mercado altamente competitivo. La comunicación con sus públicos es clave para la construcción de la imagen de empresa. El establecer una estrategia basada en los principios de coherencia, consistencia y con un enfoque claro puede servir de gran ayuda, tal como apuntan diferentes autores, tales como Trout, Sanz de la Tajada (1996), Kotler (2000), Ries (2002), Wilson \& Gilligan (2007), o Porter (2012), entre muchos otros. En definitiva, la adecuada estrategia de comunicación es el ancla para obtener un posicionamiento comunicativo único y privilegiado. Tuenti, compañía que nació en 2006 como red social española, ha realizado una evolución singular hasta ser un operador de telefonía móvil virtual en la actualidad. Este cambio, convierte a la operadora en un objeto de estudio de interés en el ámbito académico-profesional. Razón por la que se presenta un análisis de los distintos materiales de comunicación externa entre los años 2012 al 2015. Finalmente, se reflexiona sobre los resultados.

Palabras clave: Redes sociales - Posicionamiento - Comunicación corporativa Imagen - Marca - Móvil

\section{Abstract}

Currently, companies aim to be unique and differentiate themselves in a highly competitive market. Communicating with their audiences is a key element to build the company image. A strategy based on the principles of coherence, consistency and a clear approach is needed to achieve it, as different authors point out, such as Trout, Sanz de la Tajada (1996), Kotler (2000), Ries (2002), Wilson \& Gilligan (2007), or Porter (2012). In short, the adequate communication strategy is the anchor to obtain a unique and privileged communicative positioning. Tuenti, a company that was born in 2006 as a Spanish social network, has made a singular evolution to become a virtual mobile phone operator. This change makes the company an object of academic interest. An analysis of various external communication materials from 2012 to 2015 has been conducted, analyzing the results and effectiveness of the campaigns carried out.

Key Words: Social networks - Positioning - Corporate communication - Image Brand - Mobile 


\section{Introducción}

La sociedad, las empresas e incluso los ciudadanos viven en un mundo muy competitivo, en el que ocupar un lugar que permita diferenciarse del resto se ha convertido en un factor determinante. Un lugar especializado donde posicionarse como elemento único, tal y como afirma Jack Trout (2001), el autor más destacado en el este ámbito. Esto se realiza para poder triunfar en el mundo de la empresa, saturado por la concurrencia de productos y servicios, habitualmente muy pocos diferentes.

Considerando esta idea específicamente en el mundo de la comunicación organizacional, ese elemento único debe ocupar también un lugar privilegiado dentro de la mente de los públicos objetivos. Este proceso, requiere de un proceso continuado y se realiza a través de lo que se conoce como estrategia de posicionamiento comunicativo, cuyo objetivo es en última instancia ocupar un lugar referencial único y privilegiado. Para ello, la estrategia debe seguir una serie de principios que otorguen coherencia, consistencia y enfoque sobre la percepción del producto o servicio, la marca o empresa u organización en cuestión. Por supuesto, es sabido de los alcances limitados, según los principios contemporáneos de la psicología aplicada a la comunicación persuasiva, por lo que respecta al cambio de percepciones entre los sujetos sobre un objeto psicológico dado (Petty y Priester, 1996), por lo que estos elementos cobran aún más importancia en una estrategia de posicionamiento comunicativo a lo largo del tiempo.

Los principios de coherencia, consistencia y enfoque claro de la percepción de los objetivos y valores, han sido señalados ampliamente por parte de diferentes autores, además de Trout, como Sanz de la Tajada (1996), Kotler (2000), Ries (2002), Wilson \& Gilligan (2007), o Porter (2012), entre muchos otros. Todos ellos señalan que una estrategia de posicionamiento comunicativo debe estar basada en estos principios para conseguir obtener a lo largo del tiempo una posición en la mente del cliente prospecto y situar al producto o marca como opción única y diferenciada respecto a la competencia, lo que contribuye netamente a la eficacia en el cumplimiento de los objetivos de comunicación persuasiva. 


\section{ARTÍCULOS DE INVESTIGACIÓN}

Trout recomendaba llegar primero a la mente del público objetivo para conseguir una posición diferenciada respecto a la competencia. Y todo ese proceso se conseguía con una estrategia de posicionamiento basada en ofrecer una idea simplificada (Trout, 2001), con un argumento sólido, dónde no se cambie de opinión de manera constante y que sea coherente a lo largo del tiempo. A través de esta estrategia, la empresa tendría que conseguir que el cliente conozca y recuerde el producto a través de las relaciones públicas primero y después a través de la publicidad. Así, con el paso del tiempo, se conseguirá ofrecer un producto especializado y una idea diferente basada en un único atributo.

Si no se sigue ese proceso, se cometerá el error de la extensión de línea basada en una estrategia de pérdida de percepción, que choca de manera frontal con la idea de elegir un atributo diferenciador para colocarse en la mente de un público objetivo.

Según los principios teóricos mencionados, para que una empresa consiga posicionarse en la mente del público de interés concreto, es necesario trabajar una estrategia de posicionamiento basada en diferentes atributos. Atributos que sean diferenciadores y que ofrezcan una idea única y especial de la empresa y/o producto en cuestión. Las operaciones de posicionamiento deben llenarse de mensajes diferentes, simples, innovadores, obvios y depurados para poder internarse en la mente del cliente prospecto (Ries, 2002).

Para conseguir, por tanto, que la empresa o producto en cuestión se diferencie en términos de comunicación dentro del mundo hipercompetitivo en el que se vive, es necesario que la estrategia de posicionamiento esté basada en los siguientes principios, anunciados por Trout (2001): Simplicidad, mensajes directos y simples son lo que mejor llegan a la mente; Liderazgo, hay que ser el primero en llegar a la mente del cliente; Dualidad, si no se puede ser el primero, hay que serlo en la siguiente categoría; Ilusión, para provocar el deseo de compra; Debilidad y fortaleza, estudiar al competidor de cerca; Enfoque y coherencia, no perder la perspectiva y los valores de la marca y evitar la extensión de línea basada en la hiper-adaptación a las tendencias del mercado; Nomenclatura, nombre de marca que funcione de oído; Percepción, respetar las ideas interiorizadas en la 
mente del consumidor y a partir de ahí generar la estrategia; Reposición: cuando escaseen los nichos de mercado hay que reposicionar a la competencia. El reposicionamiento es un complemento al proceso de posicionamiento; Diferenciación y especialización basadas en atributos únicos de la marca; Paciencia, la estrategia funciona a largo plazo; Consistencia, no cambiar de opinión en corto plazo; Obviedad, las palabras correctas son las más simples y las que más cerca están de los valores de la marca; Singularidad, no se puede ser todo, hay que elegir lo que mejor caracterice a la marca; y transparencia, asumir el éxito y fracaso con sinceridad.

Así pues, son numerosos los principios de posicionamiento y cada autor se centra en destacar varios de ellos, por lo que este estudio exploratorio siguiendo a Trout, Ries, Rivkin y Peralba, se ha centrado fundamentalmente en los ya mencionados principios de coherencia, consistencia y enfoque de la percepción, que son los que se consideran de manera más recurrente por parte de los diferentes autores como los principales para trabajar sobre una metodología de gestión de las percepciones.

\section{Objetivo, materiales y metodología}

A través de la realización de un estudio exploratorio, los objetivos del mismo pasan por comprobar de manera práctica si los principios básicos del posicionamiento comunicativo se habían aplicado o no a un caso concreto y real de reposicionamiento de una determinada empresa, analizando sus actividades de comunicación publicitaria y no publicitaria como elementos que contribuyen a la redefinición y gestión de la identidad corporativa de la organización.

La compañía elegida fue Tuenti, la famosa red social española que nació en el año 2006 de la mano de jóvenes emprendedores que la convirtieron en la compañía de internet más importante en España con 10 millones de usuarios activos. Tuenti consiguió en unos años ser la red social más importante y tuvo entre los más jóvenes a sus principales seguidores. 


\section{ARTÍCULOS DE INVESTIGACIÓN}

\subsection{Tuenti: el caso de estudio}

Tuenti había nacido entre universitarios pero rápidamente creció entre los adolescentes convirtiéndose en todo un icono generacional. La generación Tuenti en España se identificaba con los adolescentes usuarios intensivos de la red social que se caracterizaba por ser una red social que funcionaba por invitación, por tanto muy cerrada y privada.

Los adolescentes españoles sustituyeron el uso del correo electrónico por la mensajería interna de esta red y el envío de fotos por el servicio de subida de imágenes y etiquetado.

En 2008 se daban datos de 2 mil millones de páginas vistas al mes, lo que es una cifra que simplemente abruma comparada con otros servicios y otras webs en internet en español y que convirtieron a esta red social en un fenómeno entre los jóvenes españoles, siendo en aquel momento la web con más páginas vistas en España, incluso por delante de webs como Google. Este simple dato nos refleja su poder y que la actividad de sus usuarios era tremendamente intensiva (visitar las fotos colgadas por los amigos donde aparecían ellos, participar de eventos, etc.).

La compañía fue dando entrada a nuevos inversores y evolucionando, diversificando su producto, especialmente desde 2010 con el inicio de su servicio de telefonía móvil. A lo largo de los últimos años, especialmente entre 2012 y 2015, la compañía se fue transformando progresivamente para convertirse plenamente en un operador de telefonía móvil virtual (Cid, 2016). La red social se transformó para convertirse en una aplicación móvil que no agradó a sus fieles y la mayoría de ellos se pasaron a Facebook y a otras redes sociales así como aplicaciones de mensajería instantánea. La compañía decidió mantener la marca Tuenti, con algunas modificaciones visuales y nominales parciales en ese tiempo.

Para comprobar si los principios del posicionamiento comunicativo se aplicaron en este caso práctico concreto, se analizaron distintos materiales de comunicación externa de Tuenti en el período comprendido entre los años 2012 y 2015. En concreto, se consideraron materiales de prensa y el blog oficial de la compañía, 
considerando los titulares, primeros párrafos (leads) y párrafos finales corporativos (boiler plates) de sus notas de prensa, y sus entradas al blog durante los años ya señalados en los que el reposicionamiento de la marca fue mucho más ostensible. Además, se analizó también la publicidad audiovisual realizada por la compañía en ese mismo período. Así se estableció una comparación entre los canales de texto mencionados y los audiovisuales, en concreto con las cuatro campañas de publicidad lanzadas por Tuenti a lo largo de dicho periodo de tiempo.

De manera adicional al estudio exploratorio planteado, se tomó en consideración la información publicada, es decir lo que la prensa dijo de Tuenti, para lo que se tomaron en consideración los primeros cien impactos de noticias en Google en cada uno de los años comprendidos entre 2012 y 2015.

Con todos estos elementos de análisis, se trataba de averiguar si lo que la empresa había dicho de sí misma y había sido recogido por los medios a través de estos materiales y canales, cumplía con los principios del posicionamiento ya enunciados.

Tal y como se mencionó anteriormente, para que una marca se "tatúe" en la mente del público objetivo es necesario una estrategia de marketing y comunicación basada en la coherencia, la consistencia y el enfoque claro de los objetivos y valores de la misma. Por tanto, el presente estudio exploratorio, centrado en la comunicación publicitaria y no publicitaria exclusivamente, buscará la respuesta a la siguiente pregunta que se convierte en objetivo de investigación principal: ¿La estrategia de posicionamiento y/o reposicionamiento de comunicación de Tuenti ha respetado los principios básicos teóricos del posicionamiento?

\subsection{Tipo de investigación y diseño de la misma}

La investigación exploratoria llevada a cabo fue de carácter cualitativa y descriptiva bajo la modalidad de campo. Descriptiva porque comprende la descripción, registro, análisis, interpretación y composición de los procesos o fenómenos. Tiene por objeto la identificación y catalogación de la realidad empírica de los textos o documentos mediante la definición de categorías o clases de sus elementos. Y de 


\section{ARTÍCULOS DE INVESTIGACIÓN}

campo, en tanto que incluye la recolección de datos directamente de los sujetos investigados, o de la realidad donde ocurren los hechos, sin manipular o controlar variable alguna (Arias 2006).

El diseño utilizado fue de carácter no experimental porque las variables analizadas se tomaron en su ámbito natural, sin intervención ninguna por parte del investigador. Además, dicho diseño fue descriptivo transversal porque el estudio pretendía analizar y comparar los principios del posicionamiento seguidos por Tuenti entre 2012 y 2015 y las reglas fundamentales de la materia objeto de estudio extraídas de los principios teóricos del posicionamiento.

La muestra de análisis incluyó distintas submuestras. Por una lado, las notas de prensa publicadas por el departamento de comunicación de Tuenti desde el año 2012 al 2015. En 2012 se publicaron 28 notas de prensa, en 2013 fueron 57, en 2014 se escribieron y emitieron 32 notas y en 2015 fueron 15 . En segundo lugar, se analizarán las entradas en el blog corporativo en ese periodo de tiempo: 70 entradas en 2012, 116 en 2013, 63 en 2014 y 41 entradas al blog en 2015. Finalmente, se examinarán, los spots audiovisuales realizados por Tuenti en ese período. Se analizaron los únicos cuatro anuncios que se realizaron: El primero de ellos, "Tu vida se mide en gigas" del año 2013; El segundo anuncio se realizó en 2014 con el claim "Caos de vida maravilloso"; En 2015, "La compañía móvil que se lo cuestiona todo" y también en 2015 "Haz Phone Sharing y sonríe aunque te quedes sin móvil”.

En cuanto a los documentos escritos, tanto las notas de prensa como las entradas al blog corporativo de la entidad, contaron con sus correspondientes unidades de muestreo, de registro y de contexto. Esto es en el caso de las notas de prensa y las entradas del blog: titular, el lead (primer párrafo de la nota) y el boiler plate (información final de la nota de prensa que incluye los datos básicos de la organización).

En las campañas publicitarias se consideraron las siguientes unidades de registro: El escenario y la imagen: qué se anuncia y tratamiento visual; El tiempo: duración del spot; La palabra: el slogan; La música: género utilizado en el anuncio; El argumento; Las funciones del anuncio televisivo; El público objetivo al que se dirige; Los valores transmitidos; y las conclusiones del spot. 
Para analizar la cobertura mediática, es decir cómo los medios recogieron las noticias sobre Tuenti durante el periodo de análisis, se recopilarán los 100 primeros impactos de Google en su sección de noticias. Se consideraron los 25 primeros impactos de cada uno de los cuatro años, y se analizó cómo posicionaban los propios medios a la compañía, tras la información vertida por ésta. Para llevar a cabo esta tarea, se definieron las siguientes unidades de registro: titular y el lead de cada una de las noticias recogidas por Google en dicho periodo y como una unidad de contexto la fecha de la noticia y el enlace web de la misma para ayudar a catalogar la información.
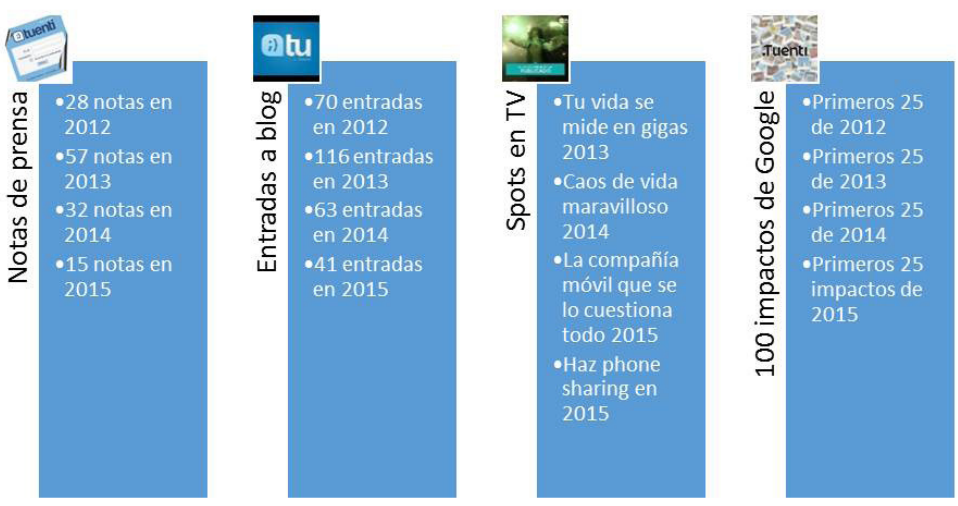

Figura 1: Submuestras de la investigación exploratoria Fuente: Elaboración propia

\subsection{Técnica de investigación}

La técnica de investigación que se empleó en este estudio exploratorio es el análisis de contenido. Siguiendo a Bardin (1996), se trata de un conjunto de técnicas de análisis de las comunicaciones tendentes a obtener unos indicadores (cuantitativos o no) por procedimientos sistemáticos y objetivos de descripción del contenido de los mensajes permitiendo la inferencia de conocimientos relativos a las condiciones de producción recepción (contexto social) de estos mensajes. El análisis de contenido propuesto fue vertical y cualitativo utilizando para ello las correspondientes fichas de análisis para cada una de las submuestras analizadas. 


\section{ARTÍCULOS DE INVESTIGACIÓN}

\section{Análisis de los resultados}

Tras la recogida de datos y el análisis de los mismos a través de las tablas de posicionamiento descritas, fue posible observar cómo había ido variando el posicionamiento proyectado por Tuenti entre los años 2012 y 2015, constatándose una amplia variedad de atributos utilizados para definir a la compañía.

En el análisis se consideraron cuatro atributos generales de compañía: móvil, operadora, tecnológica y red social. En las notas de prensa del año 2012, el número mayor de menciones fue de su carácter como compañía tecnológica (12) seguida de Tuenti Móvil (11) y Tuenti Operador (10). Tuenti Red Social solo apareció en dos ocasiones, lo que da cuenta de la pérdida de relevancia intencional de la organización a este respecto. Así pues queda patente una compañía con diferentes posicionamientos corporativos o un "Tuenti de varias cabezas" dada la poliformia de sus atributos. Uno de los primeros resultados por tanto, debido a la variedad y número de menciones, nos permite considerar que el posicionamiento de Tuenti en sus notas de prensa de 2012, no estaba plenamente enfocado ni era totalmente consistente ni coherente.

Algo similar ocurrió en el blog corporativo ese mismo año. Aunque en esta ocasión la mención mayoritaria fue para Tuenti con el atributo "móvil" (26 ocasiones), a distancia aún se encontraba Tuenti "red social", con nueve menciones. Tuenti como plataforma de comunicación (en aquel momento la compañía quiso empezar a presentar una nueva versión de su app como alternativa de mensajería) contó con siete menciones. En este canal, se observó cierta falta de consistencia entre lo que se decía en las notas de prensa y lo que se escribía en el blog corporativo, lo que se puede explicar en parte por el distinto perfil de cada canal, uno centrado en los profesionales de la información y otro en un público más amplio. De cualquier forma no se puede afirmar que exista plena coherencia, ni consistencia ni un enfoque nítido en el uso de atributos en ambos canales de comunicación.

En 2013 el posicionamiento comunicativo de la empresa objeto de estudio fue similar al de 2012 si bien fue mucho más activa cuantitativamente hablando. Durante aquel año existió mayor número de notas de prensa y también fue mayor el 
número de atributos de comunicación utilizados. La características de plataforma y multiplataforma de comunicación cobraron importancia (14 y 12 menciones respectivamente) con respecto al año anterior. La definición como compañía tecnológica siguió siendo muy relevante (27 menciones), pero el atributo que cobra mayor protagonismo, el que contó con mayor número de menciones fue Tuenti móvil (70 menciones), seguida de Tuenti 0perador (40). Tuenti como red social siguió siendo aún relevante, con 15 menciones en total. Esta cantidad y variedad de posicionamientos comunicativos de la empresa, hacen pensar de nuevo y de manera acrecentada en una posible incoherencia e inconsistencia de los mensajes, al igual que en la falta de un enfoque rotundo, nítido y transparente. Aunque el posicionamiento mayoritario es Tuenti móvil y Tuenti como operador, se sigue posicionando a Tuenti como red social, y a la vez como multiplataforma o plataforma de comunicación.

Algo similar ocurrió también en el blog ese mismo año 2013. En ese canal, aunque el atributo más mencionado tras la suma de titulares, leads y boiler plates fue el de Tuenti móvil (54 menciones), como ocurrió en 2012, destacan dos conceptos nuevos: Tuenti social messenger (16 menciones) y Tuenti como app (20 menciones), atributos que se repiten con frecuencia a lo largo del año señalado. El atributo red social solo contó con 2 menciones.

En 2014, en las notas de prensa apareció el atributo "telco" y desapareció del todo el de "red social". De nuevo, siguió existiendo una gran variedad de atributos utilizados para definir qué era Tuenti. El mayoritario fue, una vez más, móvil (48 menciones), y le siguió muy de cerca Tuenti como operador, que con 46 menciones fue el segundo más destacado. En tercer lugar con 24 menciones y empatado con el atributo telco, vuelve a aparecer el de compañía tecnológica. Tuenti multiplataforma y Tuenti plataforma de comunicación acaparan tan solo cuatro y tres menciones, respectivamente, con lo que pierden relevancia con respecto al año anterior. Una vez más, se constata la variedad y el cambio de atributos de posicionamiento en poco tiempo en aquel año 2014.

En el mismo sentido y en lo referente a las entradas de blog de ese año, siguió destacando Tuenti móvil con 40 menciones, seguido de la app de Tuenti (22) y Tuenti como operador (14). En esa ocasión, y a diferencia del año anterior, desa- 


\section{ARTÍCULOS DE INVESTIGACIÓN}

pareció el atributo Tuenti social Messenger y Tuenti multiplataforma y plataforma solo acapararon una mención, respectivamente. En este canal, desapareció Tuenti como telco y se incorporó Tuenti app que ganó en protagonismo, en un nuevo cambio de posicionamiento comunicativo.

Por lo que respecta a 2015, el análisis de las menciones de cada atributo en las notas de prensa, arrojó 11 menciones a Tuenti móvil por lo que, al contrario que en los años anteriores, este atributo perdió protagonismo relegándose a última posición. En 2015 la compañía tomó la decisión de renombrar a mediados de año su servicio de operador de telefonía, restando el apellido móvil de la marca Tuenti, lo que explica ese hecho. Tuenti operador, que podría tener el mismo significado que Tuenti móvil, acapara por ello el máximo protagonismo (21 menciones) y la compañía decide utilizar indistintamente ambos atributos.

Algo parecido ocurre con Tuenti compañía tecnológica, que vuelve a destacar con 18 menciones, y Tuenti telco, con 17. Ambos atributos pueden significar lo mismo, sin embargo la empresa los utilizó indistintamente a lo largo de las notas de prensa de 2015 .

En esta ocasión, si se tiene en cuenta que Tuenti Telco y Tuenti compañía tecnológica son conceptos equiparables, y Tuenti móvil y Tuenti operador también, podríamos afirmar que ese año se vislumbra con claridad un doble posicionamiento muy marcado o lo que hemos venido a llamar "Tuenti de dos cabezas". Por un lado, se trata de posicionar a Tuenti como una empresa de tecnología 2.0 y por el otro se define a Tuenti como un operador móvil.

En lo que se refiere a las entradas al blog corporativo de Tuenti en el año 2015, la reducción del número de atributos es palpable y aparece en un mayor número de ocasiones la marca "Tuenti", a secas, (18 menciones) sin otros atributos, debido al nuevo cambio de posicionamiento y en ese caso también de identidad visual corporativa, incluyendo el cambio de logo, llevado a cabo por la compañía como hemos señalado a mediados de ese año. Se recuerda aquí que Tuenti comenzó a denominarse ".Tuenti", y a definirse como la compañía móvil (que no operador) que se lo cuestiona todo a partir de junio de 2015. Y es por esta razón, 
que a partir de entonces Tuenti pasó a ser .Tuenti. Sin embargo, siguió estando presente el atributo de Tuenti móvil porque, como hemos señalado al referirnos a las notas de prensa, no es hasta mitad de año cuando se procedió a realizar el cambio en el posicionamiento. Tuenti app también estuvo presente pero disminuyó su peso específico de manera notable con respecto a años anteriores y solo acaparó 5 menciones.

Por tanto, se puede afirmar que el posicionamiento comunicativo de 2015 fue algo más coherente, consistente y enfocado que en años anteriores por la reducción de atributos empleados y por la concentración de las denominaciones de Tuenti y Tuenti móvil en lo que pasó a ser .Tuenti.

Teniendo en cuenta el posicionamiento trasladado en los canales de texto y en los canales audiviovisuales y realizando una comparativa entre ambos, se observó una fuerte falta de coherencia, consistencia y enfoque de la percepción. Al comparar las campañas publicitarias realizadas en formato audiovisual con los documentos escritos en el blog y en las notas de prensa, se observa cómo las campañas se centraron en posicionar a Tuenti como la compañía móvil de los jóvenes que tienen una vida basada en disfrutar con sus amigos cada vez que salen de fiesta. Una vida, que se convierte en un caos maravilloso como se especifica en el caso de 2014. Un lenguaje que no deja del todo claro que Tuenti ya no es una red social sino un operador móvil.

Sin embargo, las notas de prensa y las entradas de blog utilizadas al respecto de estas campañas, siguieron los principios del posicionamiento, con el resto de materiales escritos y se enfocaron en presentar a Tuenti con un tono corporativo, más serio, más tecnológico y no tan desenfadado como en el spot de televisión.

En lo referente a la campaña de 2014, el principio de coherencia del posicionamiento comunicativo podría cuestionarse en tanto que la compañía basó su comunicación publicitaria audiovisual en el concepto "caos de vida maravilloso", referenciando una juventud algo caótica que necesitaba el móvil para comunicarse, mientras que al mismo tiempo en sus herramientas de comunicación textual la compañía trataba de posicionarse como compañía tecnológica innovadora. 


\section{ARTÍCULOS DE INVESTIGACIÓN}

Los spots de 2015 estaban en coherencia, consistencia y enfoque tanto con las notas de prensa como con las entradas al blog corporativo, afirmando que Tuenti era una compañía móvil. Además, por primera vez en 2015 se incorporó en un spot el atributo "telco", algo coherente con la definición de Tuenti en ese momento.

Por lo que respecta al análisis de los atributos del posicionamiento comunicativo de Tuenti en medios a través del estudio de los impactos de Google, se puede afirmar que, tras el análisis de los 100 impactos de noticias recogidas por Google sobre Tuenti durante el período analizado, el posicionamiento de la compañía contó con una gran variedad de contenidos de diferentes temáticas reflejado por los medios digitales y sufrió muchos cambios a lo largo de estos cuatro años.

Siguió teniendo gran peso el pasado de Tuenti como red social, que se mantuvo durante todo el tiempo y se observó cierta confusión a la hora de mencionar a Tuenti o bien como red social o bien como operador móvil. De hecho, el año 2013 fue el más confuso en ese sentido.

Ya en 2014, Tuenti como operador y Tuenti Móvil ocupan espacios informativos cada vez más mayoritarios en la cobertura, y podemos afirmar que es en 2015, cuando el posicionamiento de Tuenti como operador móvil recogido por la prensa es unánime, si bien, a pesar de que la comunicación difundida por la compañía ya hacía tiempo que no hacía mención a Tuenti como red social, el peso de la misma en las informaciones publicadas seguía siendo relevante por el valor que como referente social Tuenti había tenido.

Se podría afirmar, por tanto, que los principios básicos del posicionamiento se hicieron más ostensibles a medida que avanzaban los años y especialmente en el último año de análisis, algo que no ocurrió en los años anteriores, dada la diversidad de atributos proyectados. 


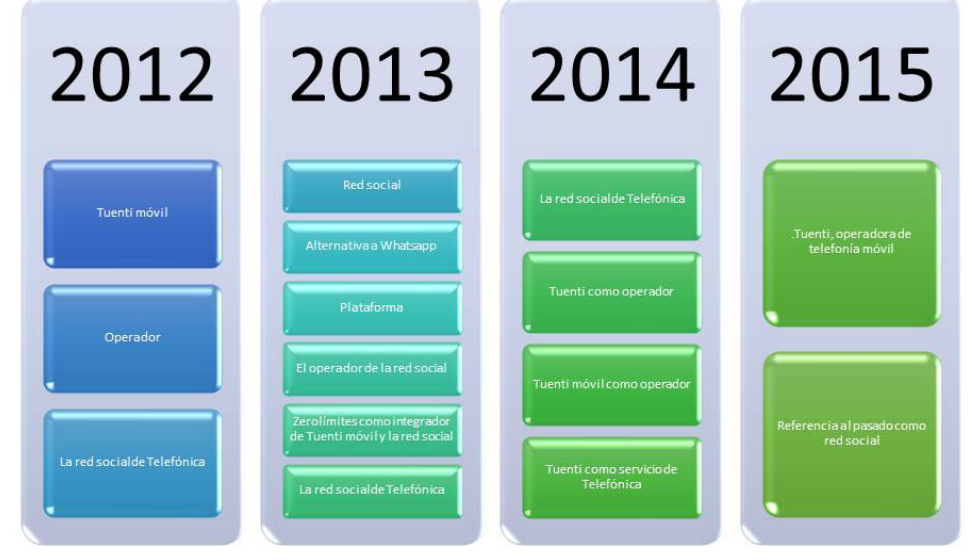

Figura 2: Tipos de posicionamiento de Tuenti en los impactos de Google Fuente: Elaboración propia

\section{Discusión}

La compañía española Tuenti nació en el año 2006 como una red social, pero cuatro años más tarde, dejó de lado progresivamente ese negocio para convertirse en un operador móvil virtual, tras la compra de la empresa por parte de Telefónica. Por tanto, en ese momento comenzó un proceso de reposicionamiento de percepciones para conseguir que el público identificara a Tuenti como un operador móvil y no como una red social.

Una vez realizado el análisis del posicionamiento comunicativo de Tuenti en tres canales de difusión publicitarios y no publicitarios: notas de prensa, entradas al blog corporativo, y la publicidad audiovisual durante los años 2012, 2013, 2014 y 2015, así como la imagen y posicionamiento recogida por los medios a través del análisis de cobertura de google, se ha podido confirmar que la compañía no empleó los mismos atributos y características para definirse a los largo del tiempo de manera consistente y reposicionarse en el mercado. Es más, a lo largo del periodo analizado la propia compañía fue variando su estrategia corporativa y de negocio y en consecuencia fue cambiando de posicionamiento comunicativo, de manera muy frecuente, lo que dificulta cualquier intento en el sentido de construir un cambio de percepciones eficaz. 


\section{ARTÍCULOS DE INVESTIGACIÓN}

Tuenti se esforzó durante cuatro años en explicar su nuevo modelo de negocio como compañía y/u operador móvil, pero no lo hizo de manera coherente y consistente, utilizando una gran variedad de atributos y sin respetar una mínima coherencia entre los mismos, de manera patente en lo que a su comunicación escrita $\mathrm{y}$ audiovisual se refiere.

Así pues, tras el estudio de caso de Tuenti, y considerando los principios enunciados por el padre del posicionamiento, Jack Trout, se puede responder claramente y de manera negativa a la pregunta de investigación planteada al principio de este trabajo.

Tras analizar las notas de prensa, las entradas en el blog corporativo y las campañas de publicidad en televisión entre los años 2012 y 2015, así como la cobertura en prensa a través de google, se puede afirmar que la estrategia de posicionamiento y/o reposicionamiento de Tuenti no ha respetado los principios básicos expresados por la teoría académica. La compañía, a tener de los resultados de este trabajo, no siguió los principios de la coherencia, consistencia y percepción anunciados por Jack Trout.

Tuenti cometió el error de la extensión de marca y de línea basada en una estrategia de pérdida de percepción, que choca de manera frontal con la idea de elegir un atributo diferenciador para colocarse en la mente del público objetivo. Además, Tuenti podría haber caído en una disfunción de su identidad (Hatch \& Schultz, 1997), motivada por la hiper-adaptación y por la falta de una idea sólida de sí misma.

Inicialmente, Tuenti lazó un operador móvil enfocado al público joven, denominado Tú, después la denominación cambió a Tuenti móvil en 2012, más tarde y en paralelo pusieron en funcionamiento el servicio de mensajería a través de su app, después se lanzaron a Latinoamérica a partir de 2014 y más tarde cambiaron de nuevo su marca y logotipo para llegar a un público más maduro, eliminando la denominación móvil. De hecho, los cambios de atributos han sido palpables a lo largo de los últimos tiempos: 


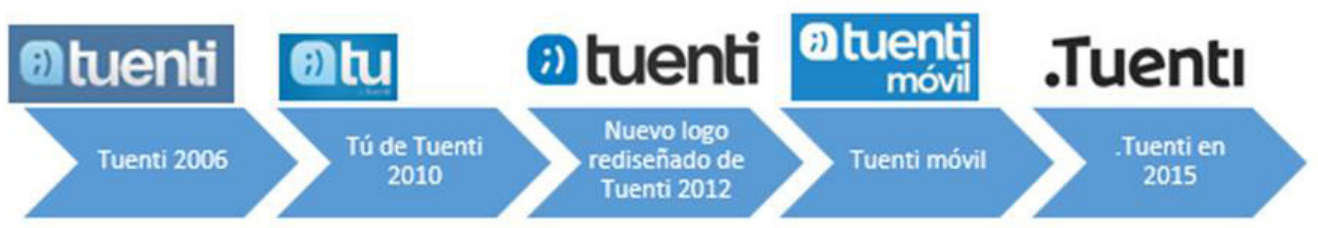

Figura 3: Evolución de la marca Tuenti

Fuente: Elaboración propia

Incluso, hubo un momento en el que Tuenti quiso ser alternativa a whatsapp como multiplataforma de comunicación y a través de las soluciones de voz.

Una estrategia muy cambiante que ha quedado plasmada en las notas de prensa $\mathrm{y}$ en las entradas al blog y que ha sido igualmente incoherente en la publicidad audiovisual, que es además la que tiene un alcance más masivo entre los públicos. No obstante, llama la atención el poco esfuerzo realizado en materia de comunicación publicitaria para dar a conocer un cambio de posicionamiento tan importante. Las campañas fueron muy pocas y con inversiones y alcances muy limitados, y aun cuando tuvieron lugar, especialmente en 2012 y 2013, lo hicieron sin explicar el nuevo posicionamiento tecnológico, sin desambiguar realmente el posicionamiento de partida y de sobra conocido: Tuenti como red social. En la comunicación no publicitaria, este esfuerzo se notó de manera más clara, pero durante los años analizados convivieron las referencias a Tuenti como red social, que siguieron siendo mayoritarias, con las de Tuenti como compañía tecnológica y las de Tuenti móvil como operador móvil virtual. La realidad del negocio y del producto sobre la que se sustentaba la comunicación publicitaria y no publicitaria no era igualmente tajante, con lo que consecuentemente no lo eran estos ámbitos.

En 2012 y 2013, las notas de prensa y el blog incumplieron los principios básicos del posicionamiento por la variedad de atributos utilizados para definir a Tuenti, presentando a Tuenti como una compañía doble, de dos caras (como compañía tecnológica de comunicación social y como operador móvil), a pesar de los intentos por anunciar la integración de Tuenti y Tuenti móvil, como el realizado en la entrada del blog número 131. Sin embargo, dicha integración no fue tal realmente 


\section{ARTÍCULOS DE INVESTIGACIÓN}

y la red social siguió existiendo conviviendo en paralelo al desarrollo del negocio como operador. Es más, durante un periodo de tiempo convivieron dos modelos de aplicación móvil, la red social clásica, y una nueva aplicación centrada en la mensajería instantánea que se conoció como nuevo Tuenti. Por consiguiente, una cosa es lo que dice la compañía que va a hacer y otra distinta es lo que realmente hace. En este sentido, de manera evidente se incumple de manera permanente el principio de coherencia. Es más, si observamos en concreto la publicidad del año 2013, se incumplen dichos principios de manera ostensible. Las piezas no dejaban ni siquiera realmente claro que Tuenti era un operador móvil, y mucho menos centrado en la tecnología, sino que inmediatamente los elementos utilizados ubicaban al espectador en el ámbito tradicional de la red social para adolescentes. La publicidad va por un lado, y la comunicación corporativa a través de la nota de prensa y la entrada al blog va por otro. Tanto es así, que en la campaña se introduce el atributo "caos" como protagonista de la misma, que es incluso parte del claim publicitario. Algo que no suena muy coherente, teniendo en cuenta que el operador móvil de Telefónica estaba despegando y no tenía mucho sentido posicionarlo como el operador del caos, un atributo netamente juvenil, cuando además de superar su pasado adolescente quería posicionarse como una compañía tecnológica, sin que utilizara el más mínimo atributo en este sentido.

A partir del año 2014 hubo un posicionamiento aún más claro en los elementos de comunicación externa no publicitarios, incorporando por ejemplo un boiler plate en las notas de prensa más consistente enfocado en Tuenti como compañía de telecomunicaciones, aunque sin embargo, encontramos ejemplos que vuelven a subrayar la falta de consistencia, como la expansión del negocio a Latinoamérica se realizó inicialmente como red social y sin haber dejado claro cuál era su modelo de negocio en España.

La llamada extensión de línea se puede observar al renunciar a un único atributo diferenciador para posicionar a la compañía y hablar indistintamente de Tuenti móvil, Tuenti como app (aplicación móvil) o Tuenti como compañía tecnológica.

En 2015, por fin, queda aún más claro que la compañía quiere dejar de lado su pasado como red social y los materiales de prensa presentan a Tuenti como 
una compañía netamente tecnológica que apuesta por dar servicio como operador móvil. Las acciones de publicidad empezaron a cumplir con los principios del posicionamiento, pero tampoco se puede decir que la empresa siguiese los principios deseables para lograrlo de manera eficaz, y por ejemplo el claim utilizado: “la compañía móvil que se lo cuestiona todo", no era del todo claro para una estrategia de posicionamiento basada en atributos tecnológicos.

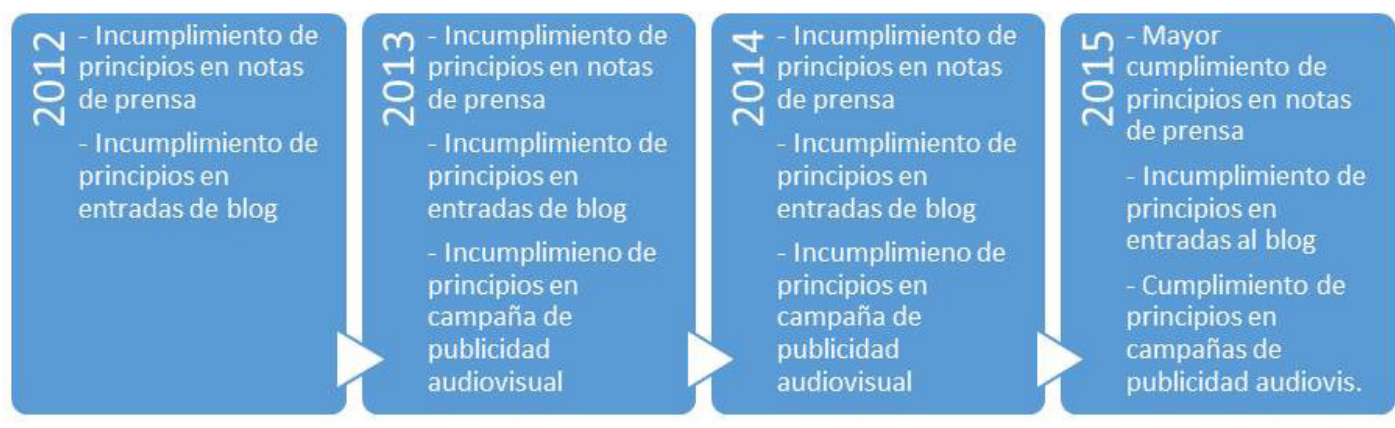

Figura 4: Incumplimiento de los principios del posicionamiento

Fuente: Elaboración propia

De manera adicional, los 100 impactos de Google analizados muestran que el pasado de Tuenti como red social seguía teniendo gran peso y que existía una cierta confusión a la hora de mencionar a Tuenti o bien como simplemente Tuenti o bien como Tuenti móvil. Parece lógico pensar que, si la empresa no ha seguido los principios recomendados, la prensa a la hora de escribir sobre Tuenti tampoco tuviera claro cómo definir a la compañía.

Tal como dice Peter Drucker (2002) la función principal de toda organización es emprender innovando y el proceso de reposicionamiento de marca se puede conseguir con el proceso de construcción de reputación de marca. Como sabemos, las percepciones se construyen a lo largo del tiempo y un cambio de las mismas no se consigue fácilmente cuando están claramente asentadas. Este es el caso de Tuenti, la notoria red social que llego a alcanzar los 10 millones de usuarios activos, ampliamente conocida en la sociedad española, y con una percepción muy definida al respecto, como para red social de adolescentes. Modificar esta percepción, explicar 


\section{ARTÍCULOS DE INVESTIGACIÓN}

un cambio de posicionamiento radical, como operador móvil, no es posible en el corto plazo si no se realiza un gran esfuerzo de comunicación al mismo nivel de notoriedad previo, y menos aún si no se respetan los principios básicos del posicionamiento: la coherencia, la consistencia y el enfoque de la percepción. Este caso de estudio, así lo atestigua.

\section{Referencias}

Arias, Fidias G. (2006). Proyecto de Investigación: Introducción a la metodología científica ( $5^{\mathrm{a}}$ ed.). Caracas: Episteme.

Bardin, L. (1996 2a e). Análisis de contenido. Madrid: Akal.

Cid, M. (2016). Tuenti y su paso de red social a 0MV ¿éxito o fracaso? Xatakamovil. Recuperado de http://www.xatakamovil.com/omvs/tuenti-y-su-paso-de-redsocial-a-omv-exito-o-fracaso (última fecha de entrada 15/02/16).

Dans, E. (2016). La evolución de Tuenti. Enrique Edans. Recuperado de http:// www.enriquedans.com/2016/02/la-evolucion-de-tuenti.html (Última fecha de entrada 15/02/16).

Drucker, P. (2002). El Management, Escritos Fundamentales. Buenos Aires: Editorial Sudamericana.

Hatch, M. y Schultz M. (1997). Relations between Organizational Culture, Identity and Image. European Journal of Marketing, 31 (5/6), pp. 356-365.

Krippendorff, K. (1990). Metodología de análisis de contenido. Teoría y Práctica. Barcelona: Piados Comunicación.

Kotler, P. (2000). Introducción al marketing (2a ed. europea, reimp. ed.). Madrid: Prentice Hall.

Operadores Móviles Virtuales: Estrategias y 0portunidades (2005). DMR Consulting, S.L. Madrid: Colegio oficial de ingenieros de telecomunicación. Recuperado de http://www.coit.es/publicaciones/bit/bit159/18-25.pdf (Última fecha de entrada 05/02/2016).

Petty R.E. \& Priester, J.R. (1996). The gradual threshold model of ambivalence: Relating the positive and negative bases of attitudes to subjective ambivalence. Journal of Personality and social Psychology, 71, 431-449.

Porter, M. E. (2012). Estrategia competitiva: Técnicas para el análisis de los sectores industriales y de la competencia. Madrid: Pirámide. 
Ries, A. (2002). Posicionamiento: La batalla por su mente. México: McGraw-Hill. Sanz de la Tajada, L. Á. (1996). Auditoria de la imagen de la empresa: Métodos y técnicas de estudio de la imagen. Madrid: Síntesis.

Soy de imago (2015). Tuenti cambia su imagen corporativa: renovarse o morir. Soy de Imago. Recuperado de http://soydeimago.com/blog/tuenti-cambia-suimagen-corporativa-renovarse-0-morir/ (Última fecha de entrada 31/03/2016).

Trout, J. (2001). Diferenciarse o morir: Cómo sobrevivir en un entorno competitivo de alto riesgo. Madrid: McGraw-Hill.

CID

Wilson, R., \& Gilligan, C. (2007). Strategic marketing management: Planning, implementation, and control. London: Elsevier. 